\title{
Synthesis of Boroxine and Dioxaborole Covalent Organic Frame- works via Transesterification and Metathesis of Pinacol Boronates
}

\author{
Ehsan Hamzehpoor, Antranik Jonderian, Eric McCalla, Dmitrii F. Perepichka*
}

ABSTRACT: Boroxine and dioxaborole are the first and some of the most studied synthons of Covalent Organic Frameworks (COFs). Despite their wide application in the design of functional COFs over the last 15 years, their synthesis still relies on the original Yaghi's condensation of boronic acids (with itself or with polyfunctional catechols), some of which are difficult to prepare, poorly soluble, or unstable in the presence of water. Here we propose a new synthetic approach to boroxine COFs (based on transesterification of pinacol aryl boronates (ArBpin) with methyl boronic acid (MBA) and dioxaborole COFs (through the metathesis of pinacol boronates with methylboryl-protected catechols). The ArBpin and MBA-protected catechols are easy to purify, highly soluble, and bench-stable. Furthermore, kinetic analysis of the two model reactions reveals high reversibility (Keq 1) and facile control over the equilibrium. Unlike the conventional condensation which eliminates water by-products, the by-product of the metathesis (MBA pinacolate) allows for easy kinetic measurements of the COF formation by conventional ${ }^{1} \mathrm{H}$ NMR. We show the generality of this approach by synthesis of seven known boroxine/dioxaborole COFs whose crystallinity is better or equal to those reported by conventional condensation. We also apply metathesis polymerization to obtain two new COFs, Py4THB and B2HHTP, whose synthesis was previously precluded by their insolubility and hydrolytic instability, respectively, of the boronic acid precursors.

\section{INTRODUCTION}

Since the 2005 pioneering work of Yaghi and co-workers, crystalline two- and three-dimensional (2D/3D) porous covalent solids - Covalent Organic Frameworks (COFs) -have emerged as a unique class of organic materials with many attractive properties and applications including gas storage and separation, catalysis, sensing, optoelectronics, energy storage, etc. ${ }^{1-5}$ The wide design space of molecular building blocks provides an infinite spectrum of possible topologies, pore sizes, chemical, and physical properties in the obtained framworks. ${ }^{5}$ Achieving the crystalline order in COFs relies on reversible (dynamic) covalent bonding between their constituent building blocks, which allows the system to self-assemble into a minimum-energy topology, via error-correction mechanisms. Boroxine and dioxaborole are the first explored synthons that endow such reversibility by condensation of di/tri-functional boronic acid with itself or with polyfunctional catechols, respectively. ${ }^{1}$ Despite the limited hydrolytic stability the highly dynamic nature of the boroxine and dioxaborole links makes them very attractive synthons in the design of COFs with excellent structural order (including COF single crystals ${ }^{6}$ ) and many optoelectronically functional COFs have been synthesized by condensation of areneboronic acids. ${ }^{7-11}$ Notably, despite 15 years of extensive exploration of such COFs, only a few chemical modifications of the original synthetic approach ${ }^{1}$ have been reported. ${ }^{12-13}$

In both boroxine and dioxaborole synthesis water is produced upon condensation, thus achieving anhydrous conditions during COF formation is impractical. This potentially limits the scope of the accessible boronic acids due to their protodeborylation in presence of water. ${ }^{14}$ Many polycyclic aromatic di/tri-boronic acids are rather insoluble, which complicates their purification and limits their reactivity in the COF synthesis. On the other hand, most pinacol boronate esters are readily soluble, stable, and can be easily purified by chromatography, sublimation, or recrystallization (which is essential for producing high-quality COFs).${ }^{15}$ The synthetic access to most multifunctional arylboronic acids relies on the deprotection of the corresponding pinacol esters (Bpin). ${ }^{16} \mathrm{How}$ ever, the latter can be a surprisingly challenging task, both kinetically (due to steric bulkiness) and thermodynamically (due to the difunctional nature of pinacol). ${ }^{17}$ The standard deprotection approaches include strong Lewis acids $\left(\mathrm{BCl}_{3}\right),{ }^{18}$ oxidants $\left(\mathrm{NaIO}_{4}\right),{ }^{19}$ and reductants $\left(\mathrm{LiAlH}_{4}\right)^{20}$ which limit the scope of the building blocks that are stable in those conditions. In the case of dioxaborole COFs, a similar problem is presented for catechol building blocks which are often sparingly soluble, difficult to purify, and prone to oxidation by air.

To address this problem, Knochel, Bein, and co-workers used a two-step process where the pinacol ester of benzothiadiazolbisbenzeneboronic acid is first deprotected via $\mathrm{HCl}$ hydrolysis and then reacted in the same pot with 2,3,6,7,10,11-hexahydroxytriphenylene (HHTP) to afford a dioxaborole COF. ${ }^{13}$ However, the harsh reaction conditions required for the deprotection $\left(180^{\circ} \mathrm{C}\right.$, microwave $)$ limit the utility of this method. Also, Dichtel and co-workers have used an acetal protecting group to mitigate the challenge of oxidative instability/insolubility of multifunctional catechols, which allowed them to introduce large phthalocyanine nodes in the COF. ${ }^{12}$

Scheme 1. Conventional vs transesterification/metathesis synthesis of (a) boroxine (b) dioxaborole COFs.

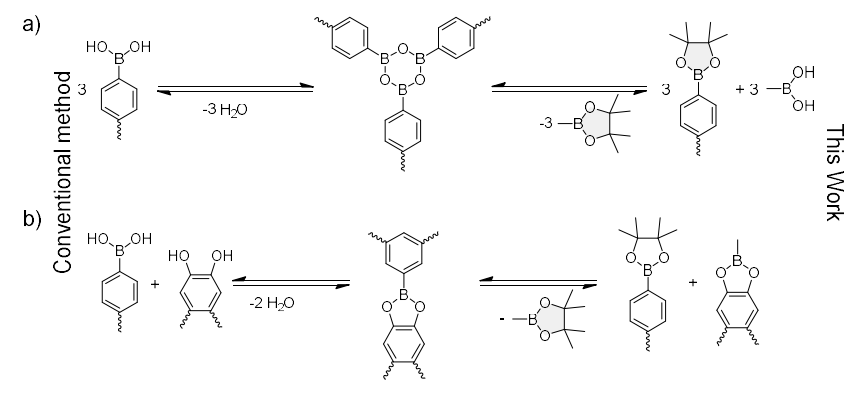



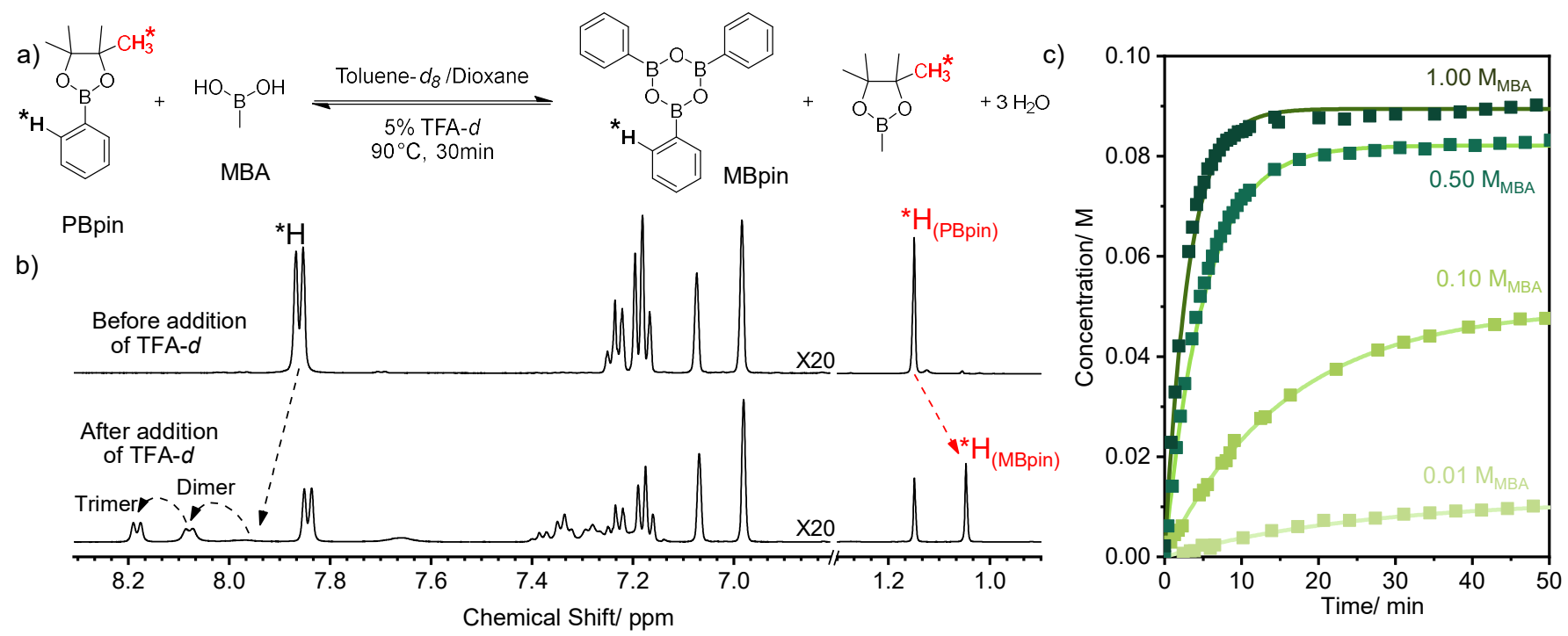

Figure 1. (a) Reaction scheme and (b) ${ }^{1} \mathrm{H}$ NMR spectra (toluene- $d 8 /$ dioxane $1: 1,90^{\circ} \mathrm{C}$ ) of PBpin $(0.1 \mathrm{M})$ and MBA $(0.1 \mathrm{M})$ before and $1 \mathrm{~h}$ after addition of TFA ( 5 vol\%); (c) corresponding kinetic plots of transesterification at different initial concentrations of MBA (scatter plots, quantified using $\delta_{\mathrm{MBpin}}=1.06 \mathrm{ppm}$ and $\delta_{\mathrm{PBpin}}=1.16 \mathrm{ppm}$ signals) and fitting kinetics curves (solid lines).

Here, we propose a new approach for the synthesis of COFs directly from esters, using transesterification with methaneboronic acid (MBA) and metathesis with MBA-protected catechols (Scheme 1). Using this strategy, we synthesized nine different boroxine and dioxaborole COFs from polyfunctional aryl-Bpins and MBA-protected 2,3,6,7,10,11-hexahydroxytriphenylene (MBA-HHTP) and 1,2,4,5-tetrahydroxybenzene (MBA-THB ). This method opens access to highly stable boroxine and dioxaborole COFs based on chemically sensitive and poorly soluble building blocks.

\section{RESULTS AND DISCUSSION}

Recently Hinkes and Klien have reported a convenient deprotection of pinacol boronate esters via their transesterification with MBA catalyzed by trifluoroacetic acid (TFA) or by collidine base. ${ }^{21}$ We hypothesized that applying this approach to multidentate aryl-Bpins may result in their in-situ polymerization directly resulting in the formation of COFs, avoiding the cumbersome deprotection step. As the reversibility/dynamic nature of the reaction is the key for COF crystallinity, we have first studied the kinetics of the model pinacol boronate PBpin deprotection with MBA in the presence of 5 vol\% TFA by NMR (Figure 1a). The reaction medium (toluene- $d_{\delta} /$ dioxane) was chosen to approximate the conditions of the solvothermal COF synthesis. ${ }^{1}$ At $90{ }^{\circ} \mathrm{C}$ and $1 \mathrm{M}$ concentration of MBA, the transesterification reaches an equilibrium in $<30$ minutes. Examining the reaction kinetics at different starting concentrations, we show that the equilibrium constant is close to unity $\left(K_{\text {eq }}=1.08\right.$ $\pm 0.10)$, and the rate constants of the forward and reverse reactions are almost identical $\left(\mathrm{k}_{\mathrm{f}} \sim \mathrm{k}_{\mathrm{r}}=5.2 \pm 0.5 \times 10^{-3} \mathrm{M}^{-1} \mathrm{~s}^{-1}\right.$ at $90^{\circ} \mathrm{C}$ and $\sim 0.13 \times 10$ ${ }^{3} \mathrm{M}^{-1} \mathrm{~s}^{-1}$ at $25{ }^{\circ} \mathrm{C}$, Figures $\left.\mathrm{S} 1,3\right)$. The deprotection is accompanied by simultaneous condensation of the resulting phenylboronic acid into triphenylboroxine, with a comparable rate (Figure 1c).

Subjecting a mixture of 1,4-di(pinacolboryl)benzene (PDBpin) and MBA to standard solvothermal conditions (mesitylene/dioxane, $120^{\circ} \mathrm{C}$ ) in the presence of TFA results in boroxine-linked COF- $1_{\mathrm{T}}(\mathrm{T}$ for transesterification) as a white powder (Figure 2). The yield of polymerization of PDBpin in a sealed ampule is controlled by equilibrium and varies from $68 \%$ with an equimolar amount of MBA to nearly quantitative (99\%) with 20 eq. excess of MBA (Table S1). The equilibrium can also be shifted by performing the reaction in an open flask while distilling off the MBpin by-product, which was used to prepare crystalline COF- $1_{\mathrm{T}}$ on a larger scale using an equimolar amount of MBA (see SI).

The spectroscopic and diffraction data of as-prepared COF- $1_{\mathrm{T}}$ are identical to those of COF-1 prepared from benzene-1,4-diboronic acid. ${ }^{1}$ The FTIR spectroscopy shows the absence of the $\mathrm{CH}_{\text {pinacol }}$ and $\mathrm{OH}$ stretch (ca. 2980, $3275 \mathrm{~cm}^{-1}$ ) and the emergence of a new strong peak at 696 $\mathrm{cm}^{-1}\left(\mathrm{~B}_{3} \mathrm{O}_{3}\right.$ ring out of plane bending $\left.{ }^{22}\right)$ indicating the successful deprotection of the PDBpin and the complete condensation of the resulting boronic acid into boroxine (Figure S7).

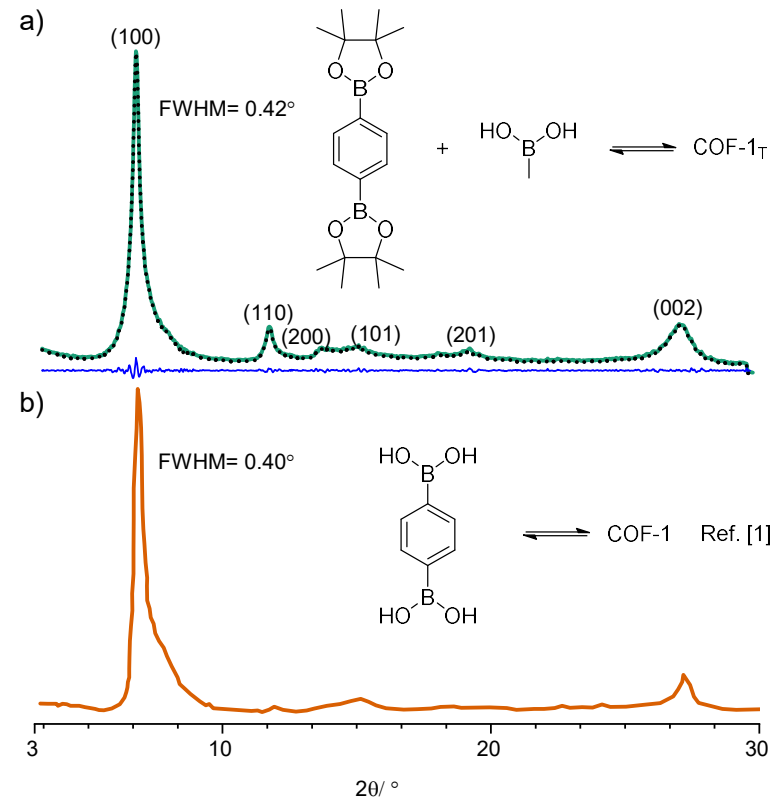

Figure 2. PXRD of activated COF- $1_{\mathrm{T}}$ (green) with Pawley refinement (dotted black, residual in blue) and of COF-1 synthesized by the conventional method (orange; replotted from ref. 1). 

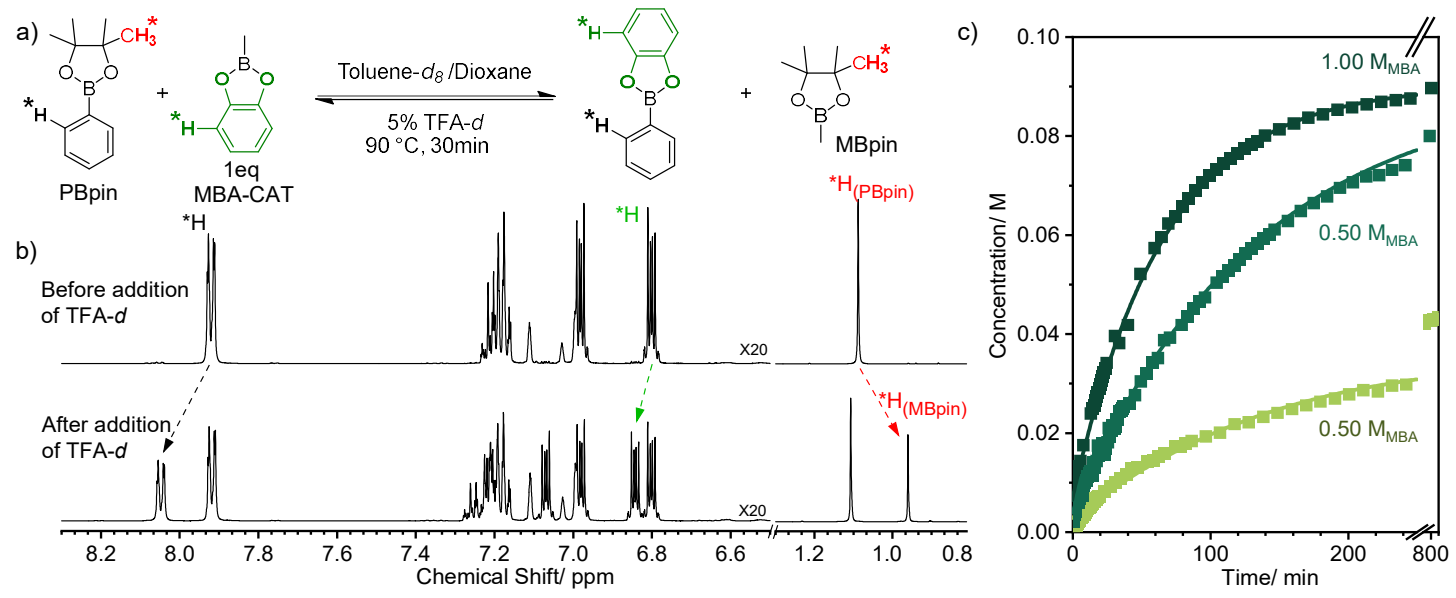

Figure 3. (a) Reaction scheme and (b) ${ }^{1} \mathrm{H}$ NMR spectra (toluene- $d 8$ /dioxane $1: 1,90^{\circ} \mathrm{C}$ ) of PBpin $(0.1 \mathrm{M})$ and MBA-CAT (0.1 M) before and $5 \mathrm{~h}$ after addition of TFA ( $5 \mathrm{vol} \%)$; (c) corresponding kinetic plots of metathesis at different initial concentrations of MBA-CAT (scatter plots, quantified using $\delta_{\mathrm{MBpin}}=1.06 \mathrm{ppm}$ and $\delta_{\mathrm{PBpin}}=1.16 \mathrm{ppm}$ signals) and fitting kinetics curves (solid lines).

The PXRD pattern of the activated $\left(180^{\circ} \mathrm{C}, 0.1 \mathrm{mbar}, 12 \mathrm{~h}\right) \mathrm{COF}-1_{\mathrm{T}}$ displayed the characteristic ${ }^{1}$ diffraction pattern with peaks at $2 \theta=6.8^{\circ}$, $11.7^{\circ}, 13.7^{\circ}, 15.0^{\circ}, 19.2^{\circ}$ and $27.1^{\circ}$ (Figure 2). The Pawley refinement (Table S8) of the diffraction pattern of COF- $1_{\mathrm{T}}$ indicates the same unit cell and very similar crystallinity $\left(\mathrm{FWHM}=0.4^{\circ}\right)$ with the published COF-1. ${ }^{1}$ The Brunauer-Emmett-Teller (BET) surface area of the activated COF-1 $\mathrm{T}_{\mathrm{T}}\left(794 \mathrm{~m}^{2} \mathrm{~g}^{-1}\right.$, Figure S10) is close to the higher end of reported surface areas for COF-1 $\left(690-812 \mathrm{~m}^{2} \mathrm{~g}^{-1}\right) .^{1,23}$

When the in-situ deprotection/polymerization is carried out in the presence of multifunctional catechols, the reaction directly produces dioxaborole-linked COFs. Thus, PDBpin in the presence of HHTP resulted in COF- $5_{\mathrm{T}}$ with diffraction and spectroscopic characteristics consistent with those previously reported for COF-5 obtained from benzene-1,4diboronic acid (Figures S13, S14). ${ }^{1}$ We note that oxidative instability and low solubility of HHTP and most other polyfunctional catechols are a significant problem for the synthesis of high-quality dioxaborole COFs. ${ }^{12}$ Using catechol monomers inevitably leads to oxidized (colored) impurities which are detrimental for optoelectronic applications. Even when the reaction is carried out in the strictest anaerobic condition, the trace amounts of catechols trapped in the COF pores will lead to noticeable colorization upon storage. To solve this problem of catechols instability and poor solubility, we decided to explore an exchange reaction (metathesis) of MBA-protected catechols with pinacol boronate esters to access dioxaborole COFs. Unlike their catechol precursors, MBA-HHTP and MBA-THB are highly stable in ambient conditions (Figure S32), soluble in common organic solvents, and can be easily sublimed under vacuum.

The kinetic measurements on the model reaction of PBpin with MBACAT show a $\sim 20$-fold decrease of the rate constants $\left(0.26 \pm 0.02 \times 10^{-3}\right.$ $\mathrm{M}^{-1} \mathrm{~s}^{-1}$ at $90^{\circ} \mathrm{C}, 5 \% \mathrm{TFA}$, Figure $\mathrm{S} 1, \mathrm{~S} 3$ ) comparing to transesterification (Figure 1). The reaction reaches equilibrium within a few hours, with $K_{\text {eq }}=0.71 \pm 0.12$. As expected, no boronic acid/boroxine intermediates are detected during the metathesis and, in contrast to all reported boronic COFs, water is neither produced during nor required for dynamic polymerization/crystallization of the COF.

COF-5 $5_{M}$ ( for metathesis) was synthesized from MBA-HHTP and PDBpin in $77 \%$ yield only in $\sim 1 \mathrm{~h}$. Surprisingly, the reaction occurred even in the absence of the TFA catalyst, over a longer time. The FTIR spectrum of COF- $5 \mathrm{M}$ is almost indistinguishable from that of COF-5 prepared from corresponding boronic acid and catechol (Figure S13), but unlike the latter, it shows the complete absence of $\mathrm{OH}$ stretch due to $\mathrm{B}(\mathrm{OH})_{2}$ defects and end-group $\left(c a .3250 \mathrm{~cm}^{-1}\right)$ in COF- $5_{\mathrm{M}}$, indicative of a much lower density of $\mathrm{B}(\mathrm{OH})_{2}$ defects compared to the standard COF-5. ${ }^{1}$ PXRD of COF- $5_{M}$ shows same sharp diffraction peaks at $3.4^{\circ}, 6.0^{\circ}, 6.4^{\circ}, 9.1^{\circ}$ as the reported COF-5 (Figure 4a). Its BET surface area $\left(1610 \mathrm{~m}^{2} \mathrm{~g}^{-1}\right)$ and the pore size $(26 \AA$, Figure $4 \mathrm{~b})$ are essentially identical to those reported for COF-5 $\left(1590 \mathrm{~m}^{2} \mathrm{~g}^{-1} ; 26 \AA\right) .{ }^{1}$ However, COF- $5 \mathrm{M}$ displays a significantly improved optical purity comparing to COF-5 (Figure 4d). As expected from its large DFT-calculated bandgap (3.88 eV, Figure S29), COF- $5_{M}$ is colorless, while the dark-grey tint of COF-5 indicates the presence of catechol oxidation products which are also manifested in a strong EPR signal (Figure 4c). The fluorescence lifetimes of the COF- $5_{M}$ show longer decay times as compared to COF5 (Figure 4e, S28; $\tau=5.7 \pm 0.13 \mathrm{~ns}$ for COF- $5_{\mathrm{M}}, 2.3 \pm 0.19 \mathrm{~ns}$ COF-5 prepared under $\mathrm{N}_{2}$ and $3 \mathrm{~ns}$ for COF-5 reported previously) ${ }^{24}$ which can be attributed to the elimination of fluorescence-quenching impurities.
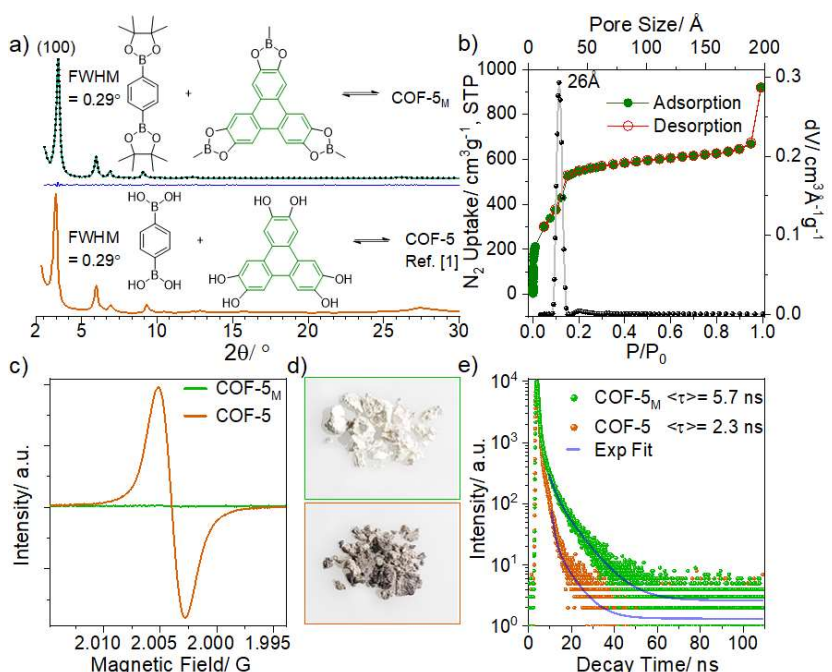

Figure 4. (a) PXRD of activated COF-5 (green) with Pawley refinement (dotted black, residual in blue, Table S8) and of COF-5 synthesized by the conventional method (orange; data replotted from ref. 1). (b) $\mathrm{N}_{2}$ adsorption isotherm for COF- $5_{\mathrm{M}}$ and corresponding pore size distribution (black). (c) EPR spectra. (d) Photographs of COF- $5 \mathrm{M}$ (top) and COF-5 (bottom). (e) Fluorescence decay curves, $\lambda_{\mathrm{ex}}=373$ $\mathrm{nm}$ and $\lambda_{\mathrm{em}}=430 \mathrm{~nm}$. 
Kinetic analysis of COF formation is important for the understanding of the growth mechanism and reaction optimization towards improved COF crystallinity/order. ${ }^{25-30}$ However, analyzing the kinetics of heterogeneous polymerization is a challenge, and only recently the experimental measurements of the kinetic of COF formation have been reported using X-ray scattering, ${ }^{30-31}$ turbidity, ${ }^{32-33}$, and PXRD/Raman spectroscopy. ${ }^{34}$ Each of these techniques possesses its unique advantages and limitations, but none has the generality/resolution of liquid NMR, as commonly employed in measuring the rate of bond formation in molecular chemistry. Since both the reactants and the byproduct (MBpin) are soluble and thus quantifiable by NMR, our metathesis polymerization allows for easy kinetic study of polymerization. Unlike turbidity which is based on monitoring the formation of $\mu \mathrm{m}$ scale COF particles and is limited to the early stages of the reaction $(<30 \%$ completion $)^{32}$, NMR screening allows the full range analysis of the kinetic $(0-100 \%)$. The kinetic measurements on the COF- $5_{M}$ formation (0.1 M PDBpin with 0.066 M MBA-HHTP) in presence of TFA (5 vol\%) at $90^{\circ} \mathrm{C}$ showed the rate constant ca. 300 times faster than when the same reaction is performed in the absence of TFA as the catalyst (Figure 5, determined from the initial rate kinetics). It is noteworthy that the rate constant of the $\mathrm{COF}$ formation is $\sim 70$ times faster than that of MBA-CAT (the model compound). This is important because the kinetics of molecular model reactions has been used in theoretical modeling of COF formation, but the differences between them are believed to be the source of discrepancies with the experimental observations. ${ }^{29}$

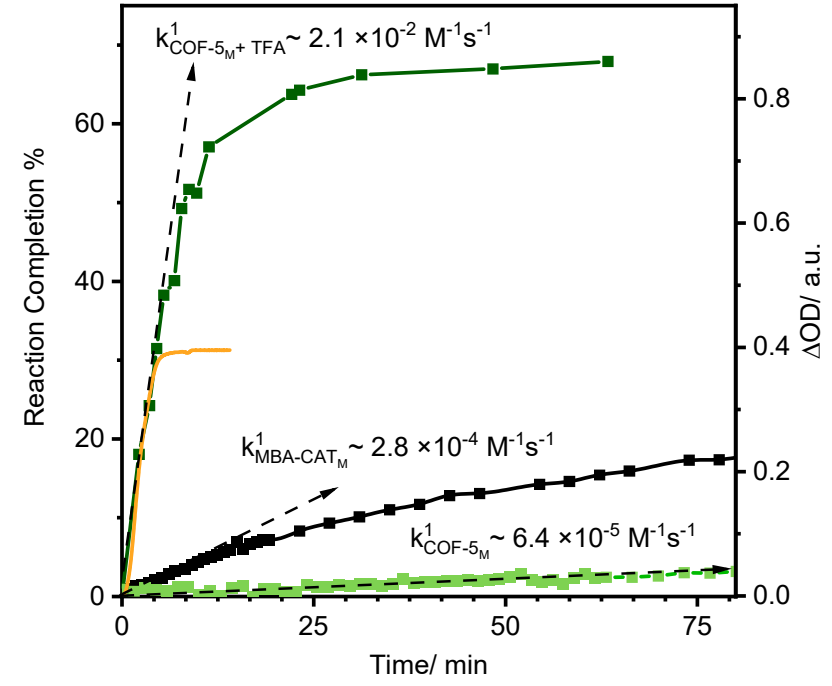

Figure 5. Kinetic plot of metathesis for COF-5 formation (0.1 M PDBpin and 0.066 M MBA-HHTP, toluene- $d_{8} /$ dioxane $1: 1,90^{\circ} \mathrm{C}$ ) in the absence of TFA (light green), and in 5 vol\% TFA (NMR: dark green, turbidity: orange) vs. metathesis of PBpin (0.1 M) + MBA-CAT (0.1 M) +5 vol\% TFA in the same conditions.

Both the transesterification and the metathesis polymerization approaches are general as demonstrated by the successful synthesis of PPyCOF, ${ }^{9}$ COF-18 $\AA$, ${ }^{35}$ COF- $6,{ }^{8}$ COF- $10,{ }^{8,}{ }^{36}$, and TP-COF ${ }^{37}$ that have been previously made from deprotected boronic acid and catechol precursors (Scheme 2). All COFs were obtained in high yields (66$79 \%)$ with crystallinity and porosities identical to the previous reports (see the SI for the characterization).

Having demonstrated the general applicability of the aryl-Bpin metathesis polymerization in the synthesis of the known COFs, we decided to apply this reaction for new COFs which would be challenging to obtain by classical condensation of boronic acids (Figure 6). The oblique Liebe lattice of Py4THB is of potential interest for materials with exotic electronic and magnetic properties. ${ }^{38-40}$ Although 1,3,6,8-pyrenetetraboronic acid is known, ${ }^{41}$ no COFs have been reported with this building block. The extremely low solubility of this compound impedes its chemical reactivity and even spectroscopic characterization. ${ }^{41}$ On the other hand, its pinacol ester, BpinPy4, is highly soluble in organic solvents and can be readily purified by chromatography and recrystallization. Metathesis of the BpinPy4 with MBA-THB produces Py4THB COF as a yellow powder in $87 \%$ yield (Figure 6a). The FTIR spectroscopy of the Py4THB shows the complete disappearance of $\mathrm{OH}$ and $\mathrm{CH}_{3 \text { (Bpin) }}\left(\mathrm{ca} .2977 \mathrm{~cm}^{-1}\right.$ ) stretching vibration bands and the emergence of the dioxaborole band at $1345 \mathrm{~cm}^{-1}$ (Figure S25). The PXRD measurement reveals characteristic diffraction peaks at $2 \theta=6.16^{\circ}(100)$, $12.35^{\circ}(200), 26.6^{\circ}(001)$ as expected for the AA stacked oblique latticemodel (Figures 6a, S30 and Table S8). The BET porosity measurement of the activated COF reveals high surface area of $822 \mathrm{~m}^{2} \mathrm{~g}^{-1}$ (Figure S26; theoretical value of $1260 \mathrm{~m}^{2} \mathrm{~g}^{-1}$ ) and average pore size of $11 \AA$ (theoretical pore size $11.4 \AA$ ).

The absence of water in the metathesis polymerization can open access to hydrolytically unstable building blocks for the synthesis of COF. Previous attempts to synthesize the B2HHTP COF by condensation of tetrahydroxydiboron with HHTP resulted in a low yield (30\%) and poor crystallinity of the product. ${ }^{42}$ We speculate that oxidative cleavage of the $\mathrm{B}-\mathrm{B}$ bond by water ${ }^{43}$ could be the reason for these discouraging results. However, metathesis polymerization of $\mathrm{B}_{2} \mathrm{Pin}_{2}$ with MBA-HHTP affords highly crystalline B2HHTP COF in a 90\% yield. The FTIR is in line with the expected changes (Figure S27) and the PXRD shows diffraction peaks at $2 \theta=4.67^{\circ}(100), 8.07^{\circ}(110), 9.22^{\circ}$ (200), $12.25^{\circ}(210), 16.71^{\circ}(310), 27.0^{\circ}(001)$ as predicted by the AA stacked hexagonal model (Figure S31 and Table S8);
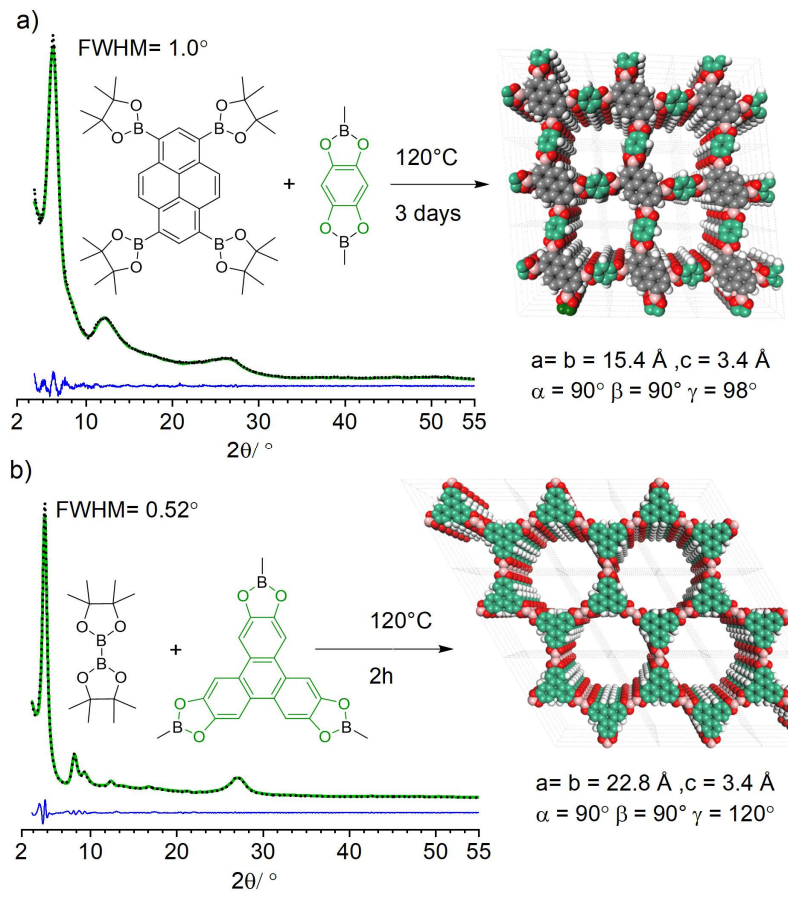

Figure 6. PXRD pattern(green) with Pawley refinement (dotted black, residual in blue, Table S8) of (a) Py4THB and (b) B2HHTP. DFT optimized (B3LYP/6-31G(d)) models with calculated unit cells shown on the right. 
Scheme 2. Scope of the COF synthesis via transesterification and metathesis reactions.

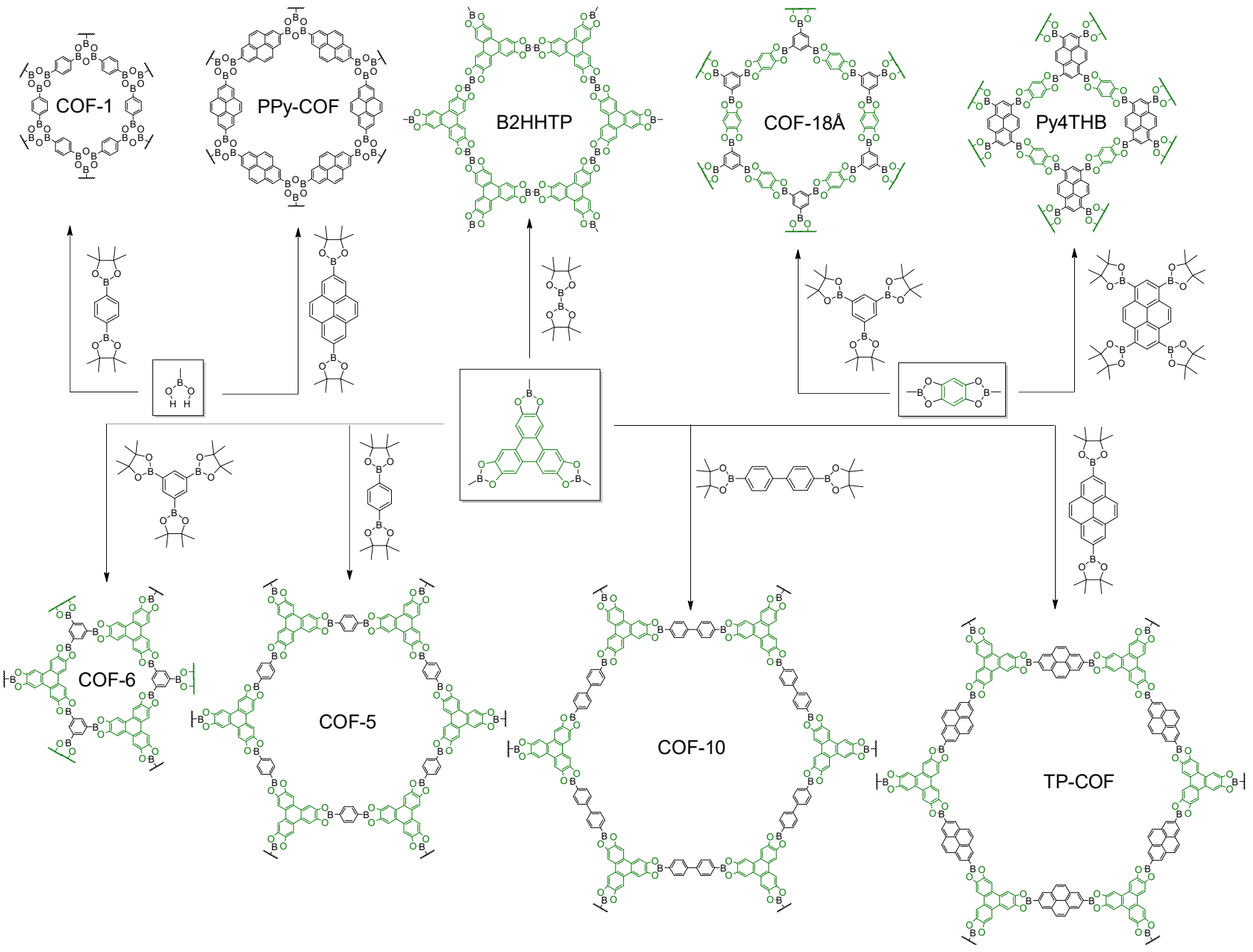

\section{CONCLUSIONS}

We reported a one-step synthesis of boroxine and dioxaborole COFs directly from pinacol-protected multidentate areneboronates using a transesterification with methylboronic acid and a new metathesis polymerization with catechol methylboronates. While preparation of such COFs from corresponding areneboronic acids is well established, our method presents noteworthy advantages for large aromatic or chemically sensitive building blocks, as it allows using highly soluble/easily characterizable and stable boronic esters instead of boronic acids and avoids the deprotection step. The dioxaborole COFs synthesized using metathesis polymerization benefit from high optical purity and low density of defects vs those synthesized by standard polycondensation, due to elimination of unprotected chemically sensitive catechols. Also, water is neither formed in the metathesis reaction nor is required to achieve crystalline COFs which opens opportunities for precise control of dynamic polymerization and provides access to sensitive areneboronic acids (which can undergo protodeborylation in presence of water). We have shown the generality of these reactions by synthesizing seven popular COFs and also applied the metathesis polymerization to obtain two new COFs (Py4THB and B2HHTP) which could not be prepared using a standard polycondensation due to the extreme insolubility and instability of the corresponding boronic acid, respectively.

\section{ASSOCIATED CONTENT}

The Supporting Information is available free of charge at [SI address] Additional details on synthesis of precursors, and COFs, XRD characterization, FT-IR, BET calculation, DFT calculations (PDF)

\section{AUTHOR INFORMATION}

Corresponding Author

Dmitrii F. Perepichka - Department of Chemistry, McGill University, Montreal, Quebec H3A OB8, Canada; orcid.org/00000003-2233-416X Email: dmitrii.perepichka@mcgill.ca

Authors

Ehsan Hamzehpoor - Department of Chemistry, McGill University, Montreal, Quebec H3A 0B8, Canada; orcid.org/0000-00025427-9444

Antranik Jonderian - Department of Chemistry, McGill University, Montreal, Quebec H3A 0B8, Canada; orcid.org/0000-0003$4357-2458$

Eric McCalla - Department of Chemistry, McGill University, Montreal, Quebec H3A 0B8, Canada; orcid.org/0000-0002-0557$608 \mathrm{X}$

Notes

The authors declare no competing financial interest. 


\section{ACKNOWLEDGMENT}

This work was supported by the U.S. Army Research Office (grant W911NF-17-1-0126) and NSERC of Canada. E.H. acknowledges MITACS and FRQNT. Authors acknowledge H. M. Titi for assistance with BET and XRD.

\section{REFERENCES}

1. Côté, A. P.; Benin, A. I.; Ockwig, N. W.; O'Keeffe, M.; Matzger, A. J.; Yaghi, O. M., Porous, Crystalline, Covalent Organic Frameworks. Science 2005, 310, 1166-1170.

2. $\quad$ Geng, K.; He, T.; Liu, R.; Dalapati, S.; Tan, K. T.; Li, Z.; Tao, S.; Gong, Y.; Jiang, Q.; Jiang, D., Covalent Organic Frameworks: Design, Synthesis, and Functions. Chem. Rev. 2020, 120, 8814-8933.

3. Liu, X.; Huang, D.; Lai, C.; Zeng, G.; Qin, L.; Wang, H.; Yi, H.; Li, B.; Liu, S.; Zhang, M., Recent advances in covalent organic frameworks (COFs) as a smart sensing material. Chem. Soc. Rev. 2019, 48, 5266-5302.

4. $\quad$ Kandambeth, S.; Dey, K.; Banerjee, R., Covalent Organic Frameworks: Chemistry beyond the Structure. J. Am. Chem. Soc. 2019, 141, 1807-1822.

5. Huang, N.; Wang, P.; Jiang, D., Covalent organic frameworks: a materials platform for structural and functional designs. Nat. Rev. Mater 2016, 1, 16068.

6. $\quad$ Evans, A. M.; Parent, L. R.; Flanders, N. C.; Bisbey, R. P.; Vitaku, E.; Kirschner, M. S.; Schaller, R. D.; Chen, L. X.; Gianneschi, N. C.; Dichtel, W. R., Seeded growth of single-crystal two-dimensional covalent organic frameworks. Science 2018, 361, 52-57.

7. Bertrand, G. H.; Michaelis, V. K.; Ong, T. C.; Griffin, R. G.; Dinca, M., Thiophene-based covalent organic frameworks. Proc. Natl. Acad. Sci. U. S. A. 2013, 110, 4923-4928.

8. $\quad$ Cote, A. P.; El-Kaderi, H. M.; Furukawa, H.; Hunt, J. R.; Yaghi, O. M., Reticular synthesis of microporous and mesoporous 2D covalent organic frameworks. J. Am. Chem. Soc. 2007, 129, 1291412915.

9. Wan, S.; Guo, J.; Kim, J.; Ihee, H.; Jiang, D., A photoconductive covalent organic framework: self-condensed arene cubes composed of eclipsed 2D polypyrene sheets for photocurrent generation. Angew. Chem. Int. Ed. Engl. 2009, 48, 5439-5442. 10. Evans, A. M.; Castano, I.; Brumberg, A.; Parent, L. R.; Corcos, A. R.; Li, R. L.; Flanders, N. C.; Gosztola, D. J.; Gianneschi, N. C.; Schaller, R. D.; Dichtel, W. R., Emissive Single-Crystalline Boroxine-Linked Colloidal Covalent Organic Frameworks. J. Am. Chem. Soc. 2019, 141, 19728-19735.

11. El-Kaderi, H. M.; Hunt, J. R.; Mendoza-Cortés, J. L.; Côté, A. P.; Taylor, R. E.; O'Keeffe, M.; Yaghi, O. M., Designed synthesis of 3D covalent organic frameworks. Science 2007, 316, 268-272.

12. Spitler, E. L.; Dichtel, W. R., Lewis acid-catalysed formation of two-dimensional phthalocyanine covalent organic frameworks. Nat. Chem. 2010, 2, 672-677.

13. Dogru, M.; Sonnauer, A.; Zimdars, S.; Döblinger, M.;

Knochel, P.; Bein, T., Facile synthesis of a mesoporous

benzothiadiazole-COF based on a transesterification process.

CrystEngComm 2013, 15, 1500-1502.

14. Cox, P. A.; Leach, A. G.; Campbell, A. D.; Lloyd-Jones, G.

C., Protodeboronation of Heteroaromatic, Vinyl, and Cyclopropyl

Boronic Acids: $\mathrm{pH}$-Rate Profiles, Autocatalysis, and

Disproportionation. J. Am. Chem. Soc. 2016, 138, 9145-9157.

15. Lennox, A. J. J.; Lloyd-Jones, G. C., Selection of boron

reagents for Suzuki-Miyaura coupling. Chem. Soc. Rev. 2014, 43, 412-

443.

16. Hall, D. G., Boronic acids: preparation, applications in organic synthesis and medicine. John Wiley \& Sons: 2006.
17. Roy, C. D.; Brown, H. C., Stability of boronic esters Structural effects on the relative rates of transesterification of 2(phenyl)-1,3,2-dioxaborolane. J. Organomet. Chem. 2007, 692, 784790.

18. Matteson, D. S.; Michnick, T. J.; Willett, R. D.; Patterson, C. D., [(1R)-1-acetamido-3-(methylthio) propyl] boronic acid and the $\mathrm{x}$ ray structure of its ethylene glycol ester. Organometallics 1989, 8, 726729.

19. Coutts, S. J.; Adams, J.; Krolikowski, D.; Snow, R. J., Two efficient methods for the cleavage of pinanediol boronate esters yielding the free boronic acids. Tetrahedron Lett 1994, 35, 5109-5112. 20. Diemer, V.; Chaumeil, H.; Defoin, A.; Carré, C., Syntheses of extreme sterically hindered 4-methoxyboronic acids. Tetrahedron 2010, 66, 918-929.

21. Hinkes, S. P. A.; Klein, C. D. P., Virtues of Volatility: A Facile Transesterification Approach to Boronic Acids. Org. Lett. 2019, 21,3048-3052.

22. Smith, M. K.; Northrop, B. H., Vibrational Properties of Boroxine Anhydride and Boronate Ester Materials: Model Systems for the Diagnostic Characterization of Covalent Organic Frameworks. Chem. Mater. 2014, 26, 3781-3795.

23. Dong, D.; Zhang, H.; Zhou, B.; Sun, Y.; Zhang, H.; Cao, M.; Li, J.; Zhou, H.; Qian, H.; Lin, Z.; Chen, H., Porous covalent organic frameworks for high transference number polymer-based electrolytes. Chem. Commun. 2019, 55, 1458-1461.

24. Flanders, N. C.; Kirschner, M. S.; Kim, P.; Fauvell, T. J.; Evans, A. M.; Helweh, W.; Spencer, A. P.; Schaller, R. D.; Dichtel, W. R.; Chen, L. X., Large Exciton Diffusion Coefficients in TwoDimensional Covalent Organic Frameworks with Different Domain Sizes Revealed by Ultrafast Exciton Dynamics. J. Am. Chem. Soc. 2020, 142, 14957-14965.

25. Koo, B.; Heden, R.; Clancy, P., Nucleation and growth of 2D covalent organic frameworks: polymerization and crystallization of COF monomers. Phys. Chem. Chem. Phys. 2017, 19, 9745-9754.

26. Nguyen, V.; Grunwald, M., Microscopic Origins of Poor Crystallinity in the Synthesis of Covalent Organic Framework COF-5. J. Am. Chem. Soc. 2018, 140, 3306-3311.

27. Li, H.; Evans, A. M.; Castano, I.; Strauss, M. J.; Dichtel, W. R.; Bredas, J. L., Nucleation-Elongation Dynamics of TwoDimensional Covalent Organic Frameworks. J. Am. Chem. Soc. 2020, 142, 1367-1374.

$28 . \quad$ Li, H.; Chavez, A. D.; Li, H.; Li, H.; Dichtel, W. R.; Bredas, J. L., Nucleation and Growth of Covalent Organic Frameworks from Solution: The Example of COF-5. J. Am. Chem. Soc. 2017, 139, 16310-16318.

29. Li, H.; Evans, A. M.; Dichtel, W. R.; Bredas, J.-L., Quantitative Description of the Lateral Growth of Two-Dimensional Covalent Organic Frameworks Reveals Self-Templation Effects. ACS Mater. Lett. 2021, 3, 398-405.

30. Castano, I.; Evans, A. M.; Li, H.; Vitaku, E.; Strauss, M. J.; Bredas, J. L.; Gianneschi, N. C.; Dichtel, W. R., Chemical Control over Nucleation and Anisotropic Growth of Two-Dimensional Covalent Organic Frameworks. ACS Cent. Sci. 2019, 5, 1892-1899.

31. Li, R. L.; Flanders, N. C.; Evans, A. M.; Ji, W.; Castano, I.; Chen, L. X.; Gianneschi, N. C.; Dichtel, W. R., Controlled growth of imine-linked two-dimensional covalent organic framework nanoparticles. Chem. Sci. 2019, 10, 3796-3801.

32. Smith, B. J.; Dichtel, W. R., Mechanistic studies of twodimensional covalent organic frameworks rapidly polymerized from initially homogenous conditions. J. Am. Chem. Soc. 2014, 136, 8783-9. 33. Smith, B. J.; Hwang, N.; Chavez, A. D.; Novotney, J. L.; Dichtel, W. R., Growth rates and water stability of $2 \mathrm{D}$ boronate ester 
covalent organic frameworks. Chem. Commun. (Camb.) 2015, 51, 7532-5.

34. Emmerling, S.; Germann, L. S.; Julien, P.; Moudrakovski, I.; Etter, M.; Friscic, T.; Dinnebier, R. E.; Lotsch, B., In Situ Monitoring of Mechanochemical Covalent Organic Framework Formation Reveals Templating Effect of Liquid Additive. ChemRxiv. Preprint. https://doi.org/10.26434/chemrxiv.12988829.v1 2020.

35. Tilford, R. W.; Gemmill, W. R.; zur Loye, H.-C.; Lavigne, J. J., Facile Synthesis of a Highly Crystalline, Covalently Linked Porous Boronate Network. Chem. Mater. 2006, 18, 5296-5301.

36. Doonan, C. J.; Tranchemontagne, D. J.; Glover, T. G.; Hunt, J. R.; Yaghi, O. M., Exceptional ammonia uptake by a covalent organic framework. Nat. Chem. 2010, 2, 235-238.

37. Wan, S.; Guo, J.; Kim, J.; Ihee, H.; Jiang, D., A belt-shaped, blue luminescent, and semiconducting covalent organic framework. Angew. Chem. Int. Ed. 2008, 47, 8826-8830.

38. Jin, E.; Asada, M.; Xu, Q.; Dalapati, S.; Addicoat, M. A.; Brady, M. A.; Xu, H.; Nakamura, T.; Heine, T.; Chen, Q.; Jiang, D., Two-dimensional sp (2) carbon-conjugated covalent organic frameworks. Science 2017, 357, 673-676.

39. Cui, B.; Zheng, X.; Wang, J.; Liu, D.; Xie, S.; Huang, B., Realization of Lieb lattice in covalent-organic frameworks with tunable topology and magnetism. Nat. Commun. 2020, 11, 66.

40. Jiang, W.; Huang, H.; Liu, F., A Lieb-like lattice in a covalent-organic framework and its Stoner ferromagnetism. Nat. Commun, 2019, 10, 1-7.

41. Wakchaure, V. C.; Ranjeesh, K. C.; Goudappagouda; Das, T.; Vanka, K.; Gonnade, R.; Babu, S. S., Mechano-responsive room temperature luminescence variations of boron conjugated pyrene in air. Chem. Commun. (Camb.) 2018, 54, 6028-6031.

42. P P, R.; Mondal, P. K.; Chopra, D., Synthesis and characterization of a $2 \mathrm{D}$ covalent organic framework (COF) of hexagonal topology using boronate linkages. J. Chem. Sci. 2018, 130, 51.

43. Wiberg, E.; Ruschmann, W., Über eine neue Borsäure (,Unterborsäure') der Formel $\mathrm{H}_{4} \mathrm{~B}_{2} \mathrm{O}_{4}$ und ihre Ester. Ber. Dtsch. Chem. Ges. 1937, 70, 1393-1402. 